\title{
INVESTIGATIONS OF MATERIAL BEHAVIOUR UNDER MONOTONIC TENSION USING A DIGITAL IMAGE CORRELATION SYSTEM
}

\author{
TADEUSZ SZYMCZAK \\ Motor Transport Institute, Centre for Material Testing, Warsaw, Poland \\ e-mail: tadeusz.szymczak@its.waw.pl
}

\begin{abstract}
The paper reports behaviour of engineering materials for different kinds of notches, i.e. having $U, V$ shapes. Their variants with respect to dimensions are illustrated in static and fatigue tests. The influence of these types of geometrical imperfections on material fatigue is presented using variations of the Wöhler curve, number of cycles to failure and the fatigue notch factor. Results of experiments conducted by the use of the Digital Image Correlation system called 4M Aramis are illustrated. Courses of tensile characteristics of the $41 \mathrm{Cr} 4$ steel, obtained by means of both techniques: extensometer and DIC are compared. They indicated that the DIC technique can be a good tool for determination of mechanical properties. The equivalent strain full-field distributions on specimens tensioned with and without imperfections up to material fracture are presented. The influence of $U$ and $V$ notches on variations of tensile curves is shown.
\end{abstract}

Keywords: geometrical imperfection, notch, extensometer, DIC, non-contact technique

\section{Introduction}

Determination of behaviour of materials under various loading types can be reached by the use of different measurement techniques. The extensometer method is the most popular approach applied in static and fatigue tests for measuring strain state components versus time. Nevertheless, this technique registeres strain variations in one direction indicated at the beginning of the experiment with respect to the load direction. These results are important for typical engineering calculations focused on the assessment of material behaviour in a one-axis coordinate system. For material examination under more complex types of loading, these data are insufficient because many materials exhibit anisotropy of mechanical properties. Moreover, observation of damage zones and strain fields is not possible and, nowadays, these material features can be followed using a modern, non-contact optical method, such as Digital Image Correlation (DIC).

The DIC method recommended for 3D measurements is a stereoscopic technique employing two CCD cameras, light sources and advanced software (GOM source). This method involves applying a special pattern represented by black dots on a grey background (Chu et al., 1985; Lord, 2009). A mathematical description of the DIC is available in the literature (Chu et al., 1985, for example). Markers are chosen by a DIC device for obtaining $x, y$ and $z$ coordinates, and are followed by the DIC system up to specimen fracture. The DIC can employ a pattern of rectangles or squares whose origins are directly selected for calculations of displacement/strain. Results are presented as full-field maps expressing distribution of strain from the test beginning up to the specimen fracture (Lord, 2009; Kamaya and Kawakubo, 2011). The maps can be compared with the FEA results (Toussaint et al., 2008; Gower and Shaw, 2010; Kamaya and Kawakubo, 2011) to validate material models or constitutive equations. The Digital Image Correlation is recommended for static and fatigue tests conducted under various types of loading. In the case of static experiments, a DIC device can be used more flexibly than in experiments on cyclic 
loading. This is due to the limited number of stages offered by the DIC software to be recorded. Therefore, a concept to follow strain variations due to cyclic loading should be formulated before testing with the use software targets or commands in $\mathrm{C}++$.

Results from various research groups show that the DIC is employed for examination of material behaviour under typical tensile and compression tests (Forster et al., 2010), fracture toughness examinations (Durif et al., 2012) and experiments focused on determination of the effects of geometrical imperfection such as notches (Kamaya and Kawakubo, 2011) and holes. Data on monotonic tension are usually represented by full-field maps expressing strain distribution and its values up to the moment of fracture appearance. The results from fracture toughness experiments present the strain distribution close to the fatigue crack and stress intensity factors.

\subsection{Specimens with notches}

Notches are defined as geometrical imperfections of structural components. Their geometry is represented by the angle between their edges, radius and depth. Many results express values of the stress concentration factor (SCF) obtained by analytical or numerical calculations. This enables the assessment of influence of the stress concentration factor on stress values appeared in the notch tip, in a one-dimensional coordinates system. The second widely used method for determination of SCF influence comes as a result of the development of Finite Element Analysis (FEA). In this case, advanced engineering software is used to solve problems caused by different kinds of geometrical discontinuities. In the case of this method, many advantages can be stated, i.e. application of various $2 \mathrm{D}$ or $3 \mathrm{D}$ elements and analysis of macro- and micro-scales. Its main drawbacks are material definitions in the elastic-plastic state, as FEA requires definition of material hardening or softening reached not only in one axial stress state, but also in a complex one. Despite the use of modern biaxial testing machines, material examination under various combination of stress components is not easy and is still being developed.

Notches are classified by many branches of industry as geometric discontinuities that can influence material behaviour and reduce lifetime of components. They appear in drive shafts, engines, car bodies, turbines and others elements as well as constructions having complicated shapes. One effect of notches, strongly related to their radius, angle, fillet at the tip and other dimensions, is that the size of these features can influence the concentration of stress/strain state components, time to fracture and failure type of the material.

Worth to distinguish round and flat specimens with macro- (Fig. 1) and micro-notches (Maruno et al., 2004; Whaley, 1964). The size of geometric discontinuities is unique for each type of specimen and depends on details of the experimental procedure. In the case of round specimens, notches are machined around the major axis of the specimen (Figs. 1 and 2) but for flat specimens, geometric discontinuities are located on one (Whaley, 1964) or both sides of the specimen (Fig. 2).
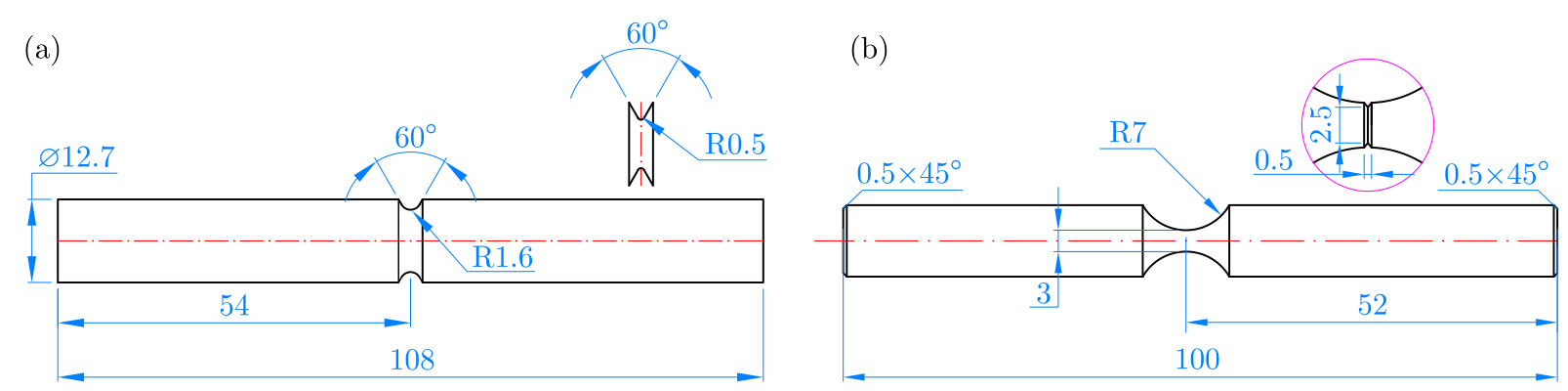

Fig. 1. Notched round specimens for fatigue testing: (a) Fatemi et al. (2004),

(b) Pluvingae and Gjonaj (2001) 

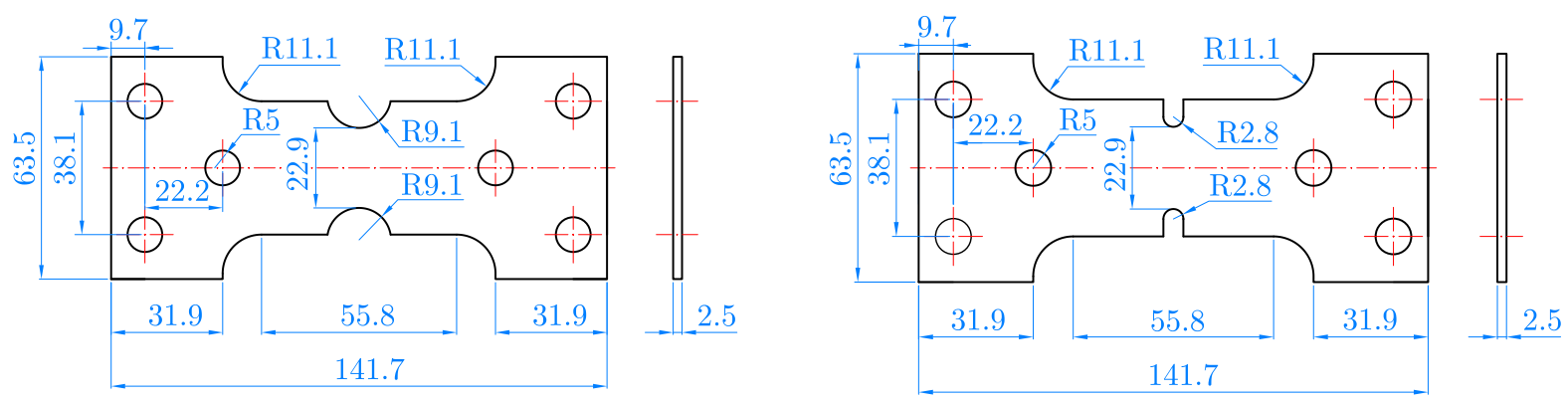

Fig. 2. Double-notched flat specimens used for examination of material behaviour under cyclic loading (Fatemi et al., 2004)

\subsection{Material behaviour due to notches and holes}

Great efforts of many research groups (Wahl et al., 1930; Mazdumar and Lawrence, 1981; Lanning et al., 1999; Milke et al., 2000; Fatemi et al., 2002, 2004; da Silva et al., 2012) are made to describe phenomenology of the influence of notches on material behaviour under various loading types up to fracture. As previously mentioned, notched specimens represent individual projects of research groups.

Experimental results from tests concentrated on determination of the influence of notches on material fatigue show the significant effect of geometric discontinues on lifetime (Figs. $3-6)$. The differences between material lifetime due to various stress levels can be equal to $80 \%$ (Fig. 3). Moreover, contrary to the results from tests on smooth specimens, the data for notched specimens indicate some difficulties in the machining of the notches. Boroński (2007) assumed that the fatigue life determined at the notch bottom was the same as the life for a smooth specimen when strains waveforms for the both specimens type were the same.

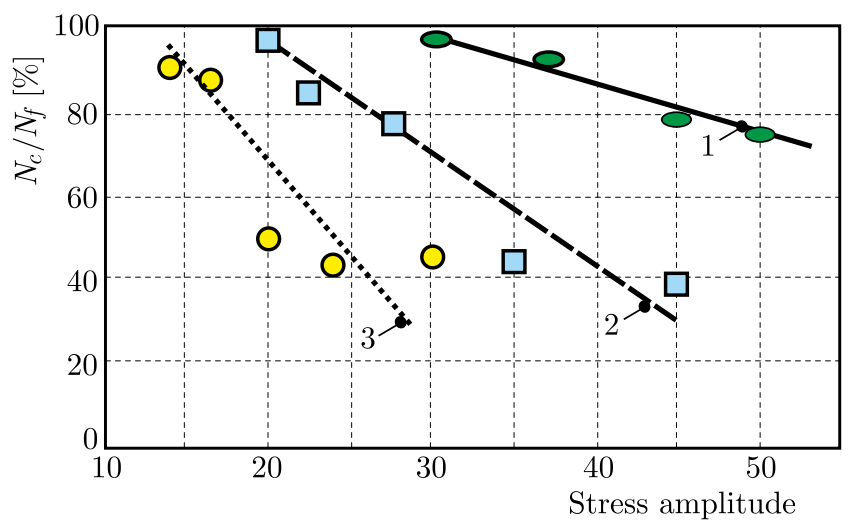

Fig. 3. The influence of notch radius on the proportion of cycle numbers to initiation of the first crack $N_{c}$ and fracture $N_{f}$ at various stress amplitudes for the specimens: 1 - un-notched, 2 - notched with radius $1.5 \mathrm{~mm}, 3$ - notched with radius $6.35 \mathrm{~mm}$; material: 24S-T4 aluminium alloy (Bennett and Weinberg, 1954)

The results presented in Fig. 4 illustrate variations of the fatigue notch factor for values of the stress ratio $R$ within a range of 0.5-1.0 due to the radius of the notch machined in cylindrical and flat specimens. In the case of flat specimens, an increase of this parameter is clearly visible. For the cylindrical specimen, the value of the fatigue notch factor achieved a constant level.

Experimental procedures are also designed to capture Wöhler curve variations at stress controlled tests, applying notches with different values of stress concentration factors (Fig. 5). These investigations are usually carried out using round or flat specimens. As can be seen in the paper by Fatemi et al. $(2002,2004)$, a great decrease of the cycle number to failure was achieved 


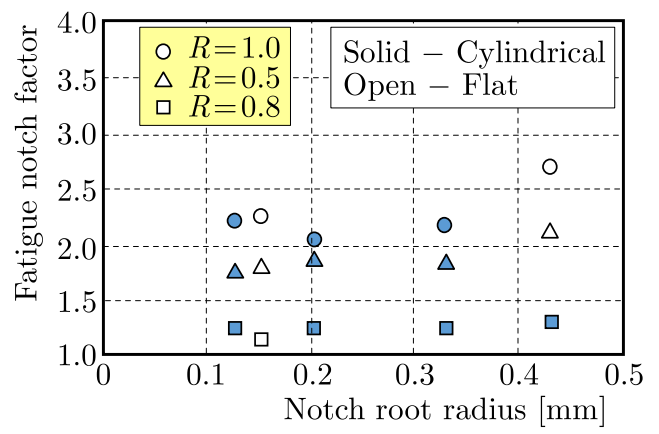

Fig. 4. Fatigue notch factor versus notch root radius for cylindrical and flat specimens with notches for a stress ratio within a range from 0.5 to 1 ; material used: Ti-6Al-4V (Lanning et al., 1999)

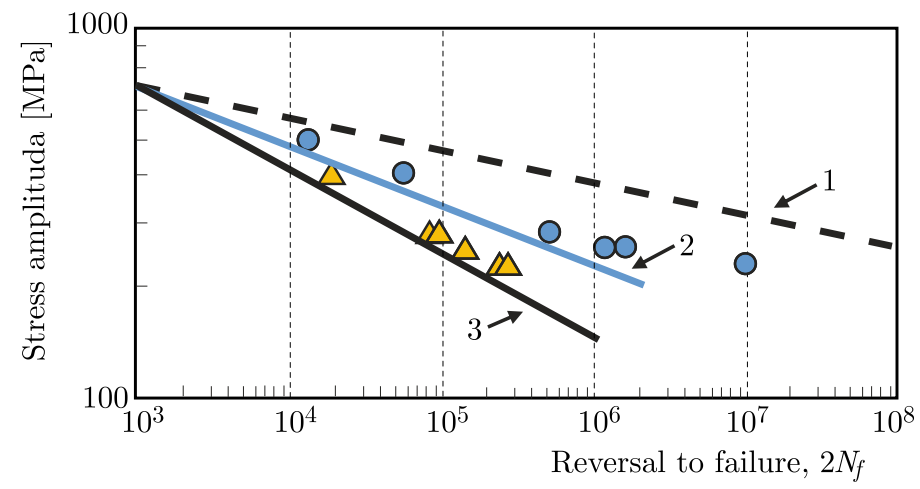

Fig. 5. Wöhler curve determined on circumferentially notched round bars for stress concentration factors equal to: 1 - 1.0, 2 - 1.787, $3-2.833$; material: 1141 medium carbon steel micro-alloyed with Vanadium (Fatemi et al., 2002, 2004)

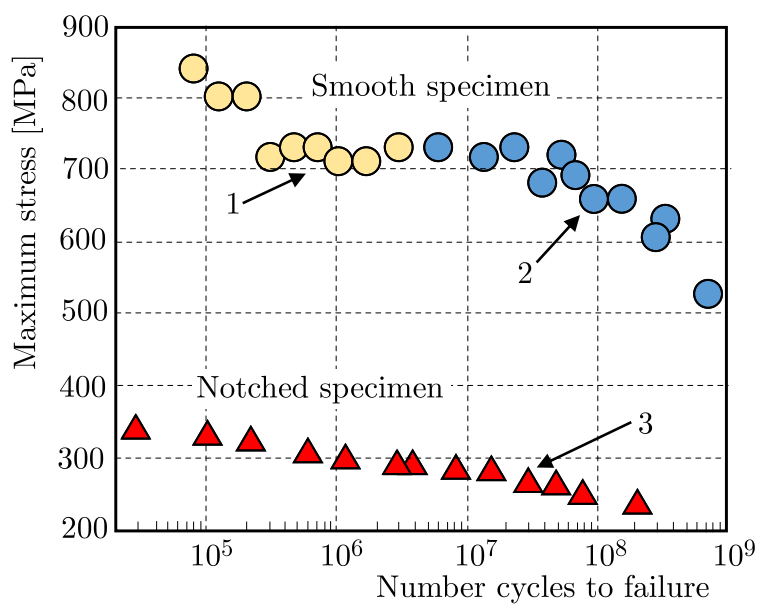

Fig. 6. Wöhler curve of $40 \mathrm{Cr}$ steel obtained for smooth and notched specimen with modes crack initiation: 1, 3 - surface, 2 - subsurface (Qian et al., 2010)

when the SCF increased three times. Distribution of experimental points from tests performed on flat specimens with the notch having a stress concentration factor equal to 1.787, expressed low agreement with a semi-logarithmic approximated function. The reason for this disagreement is usually related to the specimen machining process and their mounting in testing machines.

Other experiments were conducted to determine the zone of initiation of cracks during fatigue (Fig. 6). In that case, the stress signal was used to control the testing machine. Both smooth and notched specimen geometries were applied (Chen, 2004). The application of smooth specimens in tests performed at various stress levels allowed following the initiation and propagation of 
cracks from the surface to the subsurface. In the case of notched specimens, the cracks appeared on the surface only.

Analytical and numerical calculations are concentrated on determination of stress gradients in the tip of the notch (Siebel and Stieler, 1955; Peterson, 1959; DuQuensay et al., 1986; Filippini, 2000; Milke et al., 2000). Their magnitude are calculated using an equation representing dimensions of notches (Siebel and Stieler, 1955; Peterson, 1959; Pilkey, 1997), Neuber's theory (Neuber, 1958, 1961; Filippini, 2000; Topper et al., 1967), and proportion of stress and nominal stress in the notch (Peterson, 1959; DuQuensay et al., 1986).

In FEM analysis, the influence of notches is calculated for specimens with narrow geometrical discontinuities like $V$ or $U$ shapes (Pluvingae and Gjonaj, 2001; Andersson, 2013; da Silva et al., 2012). The maximum stress in the tip of a notch (da Silva et al., 2012; Andersson, 2013) and the stress concentration factor are parts of the results (da Silva et al., 2012) as well as an estimation of fatigue strength (Andersson, 2013).

\section{Details of the experiment}

The experimental procedure elaborated for application of the DIC system contained three stages as follows:

a) determination of the stress-strain curve, Young's modulus, yield point and ultimate tensile strength;

b) investigation of the full-field strain distribution close to $U$ and $V$ notches;

c) examination of the influence of dimensions of $U$ or $V$ notches and their interactions with material behaviour.

All tests were carried out at room temperature using servohydraulic 8802 Instron and electro-dynamic Electropuls E10000 Instron testing machines. The Aramis 4M Digital Image Correlation system was employed to follow distribution of the strain state. Before testing, the DIC device was calibrated. In the testing stages focused on determination of mechanical properties and material behaviour with the assistance of geometrical imperfections and various tensile rates, an extensometer and the 4M Aramis were used simultaneously. The assessment of the DIC system used for the determination of mechanical properties was made on the basis of a comparison of data obtained by the extensometer and by virtual tensometers defined in the DIC software.

Application of the Digital Image Correlation technique required the following stages:

a) adjustment of the distance between two cameras, its angle indicated in guidelines of technical data;

b) positioning the $4 \mathrm{M}$ Aramis with respect to the centre of the measurement zone;

c) selection of a calibration plate, which should be chosen on the basis of dimensions of the region for which the displacement is considered to be determined;

d) performing the calibration procedure by applying the plate which takes various orientations in the $3 \mathrm{D}$ coordinate system, and recording its positions;

e) mounting the specimen, having an artificial measuring zone represented by black dots stochastically arranged on the grey layer, in grips of the testing machine;

f) capturing the first photo and establishing it as the reference one for displacement determination and strain calculations.

Technical data (GOM folders) for calibration of the DIC system are delivered by the producer of this device type and contain the following features: measuring volume (height, length, width), minimum length camera support, distance ring (it enables one to change camera lens), measuring distance (from the central section of the DIC device to the centre of the measuring zone), slider 
distance (determined by two technical points on the cameras), camera angle (it determines the centre of the measuring zone), calibration object (a plate with special regular markers having determined coordinates, which should be identified by the cameras during the calibration process).

In the case of the experiments, the $4 \mathrm{M}$ Aramis was used with the following technical parameters:

- initial measuring zone determined on the basis of the specimen tested $25 \mathrm{~mm} \times 10 \mathrm{~mm} \times 3 \mathrm{~mm}$;

- calibration plate $25 \mathrm{~mm} \times 18 \mathrm{~mm}$;

- slider distance $37.5 \mathrm{~mm}$;

- camera lens $75 \mathrm{~mm}+$ slider distance;

- camera angle $25^{\circ}$;

- sampling rate 2 photos/s.

\section{Results}

Comparison of tensile curves obtained by the use of the flat specimen and the extensometer as well as the DIC system is presented in Fig. 7. The axial stress is calculated as a proportion of the axial force to the cross-section of the measurement zone. Values of the axial strain are calculated for the same base, close to $25 \mathrm{~mm}$.

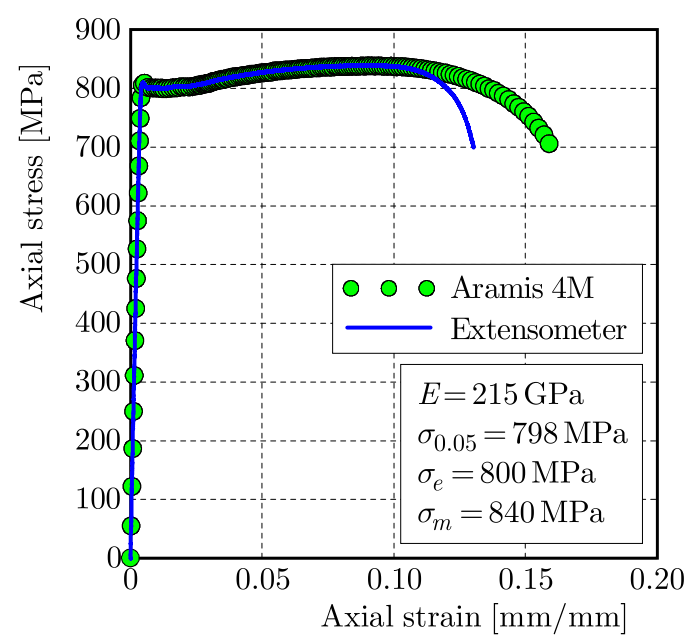

Fig. 7. Comparison of tensile characteristics determined by the use of the extensometer and Aramis 4M; material: high strength steel

A digital image correlation device and the extensometer technique have been simultaneously employed in the monotonic test. For DIC calculations, two virtual tensometers were selected to define the gauge length for determination of the axial strain. A comparison of the results from the extensometer and the DIC technique expressed high agreement in the stress-strain curves from the beginning of testing up to the ultimate tensile strength occurrence. Differences in the last section of those relationships are related to appearance of the neck, which is close to the extensometer edge.

Mechanical properties calculated on the basis of the data obtained by means of both applied techniques are listed in Fig. 7. Differences between tensile characteristics (Figs. 7 and 8) are very small, indicating the DIC system to be recommend not only for capturing strain maps (Fig. 9), but also for determination of typical mechanical parameters. 


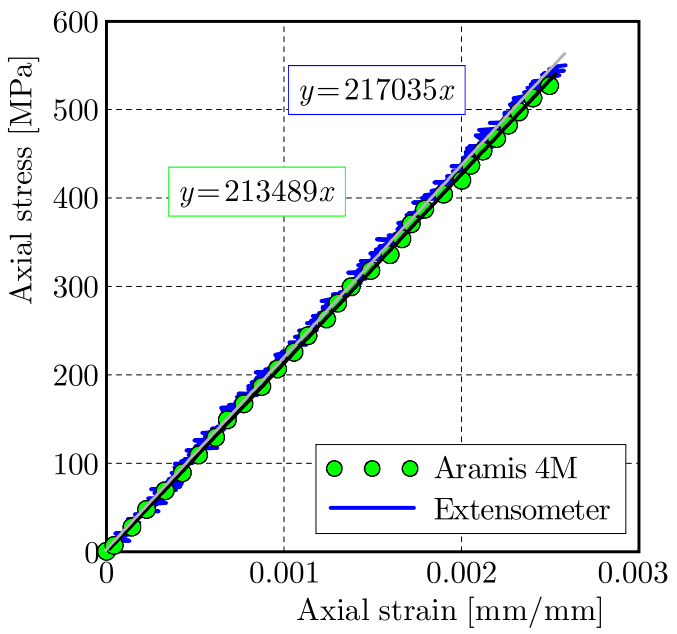

Fig. 8. Linear relationship of tensile curves illustrated in Fig. 7

(a)

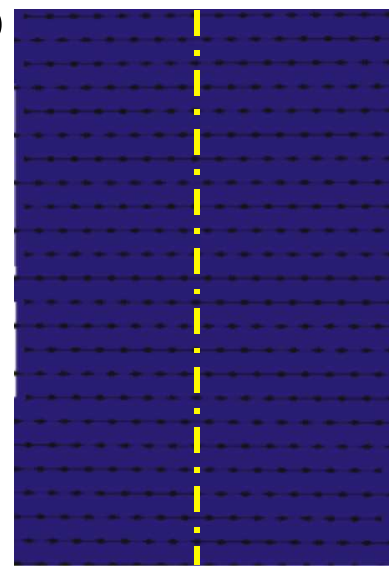

(c)

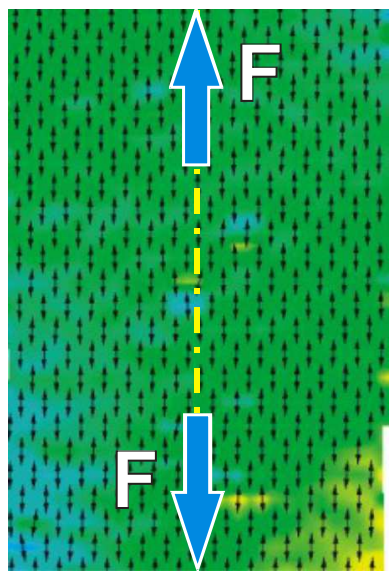

(b)

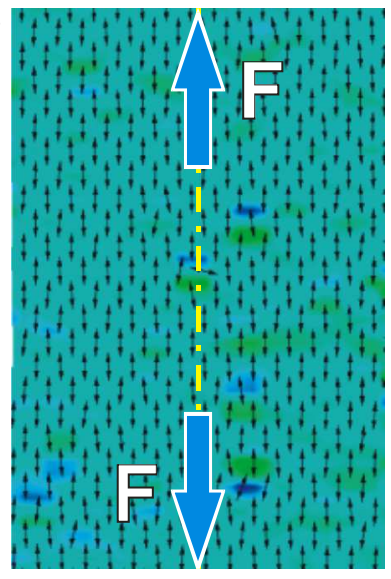

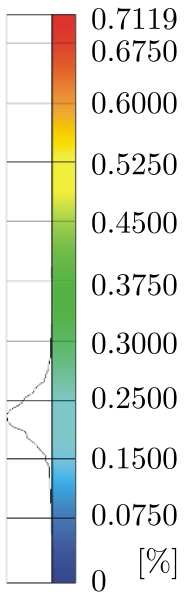

(d)

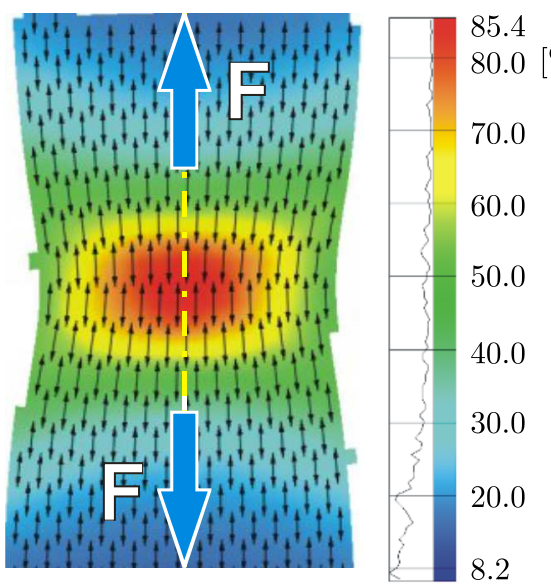

Fig. 9. Distribution of axial strain in a flat specimen under monotonic tension at various stages of the testing: (a) specimen mounted in the testing machine before the experiment, (b) at the strain-determined proportional limit, (c) at the strain-determined yield point, (d) before fracture

The main advantage of the DIC system is presented in Fig. 9, which shows variations of the axial strain distribution at various stages of tension. On the basis of these results, the damage zone can be followed up to the specimen fracture (Figs. 9b-d). It is also worth noticing that the distribution of the major strain (black vectors) at the beginning of tensile testing enables the assessment of quality mounting of a specimen in the testing machine (Fig. 9a). 
Another application of the DIC method is illustrated in Fig. 10. These figures show the equivalent strain distributions for the specimen with a $U$-shaped notch at various material states. The $4 \mathrm{M}$ Aramis system employs the equation as follows

$$
\varepsilon_{e q}=\sqrt{\frac{2}{3}\left(\varepsilon_{1 \_t r u e}^{2}+\varepsilon_{2 \_t r u e}^{2}+\varepsilon_{3 \_t r u e}^{2}\right)}
$$

where $\varepsilon_{i}$ represents the major true strains, $i=1,2,3$. The major strains are expressed by the following equation $\varepsilon_{i-t r u e}=\ln (1+\varepsilon)$, for which $\varepsilon=(\Delta l) / l_{0}$ is the engineering strain, where $l_{0}$ is gauge length, $\Delta l$-elongation. Equation (3.1) presents the stress state in the strain coordinate system. It can be reached on the basis of theory of plasticity (Westergaard, 1952; Olszak, 1965; Chen, 2004).

It should be emphasized that equation (3.1) can be used when the straining is proportional. i.e. expressed by constant ratios of $d \varepsilon_{1 \_ \text {true }} / d \varepsilon_{2 \_t r u e} / d \varepsilon_{3 \_ \text {true }}$.

Both initial images present the specimen at the beginning of the test, i.e. the referential stage with zero loading and in the elastic state (Figs. 10a,b). The elastic-plastic state, shown in Fig. 10c, expresses the equivalent strain distribution and indicates the deformation zone located close to the centres of $U$-notches as initiators of maximum strain.

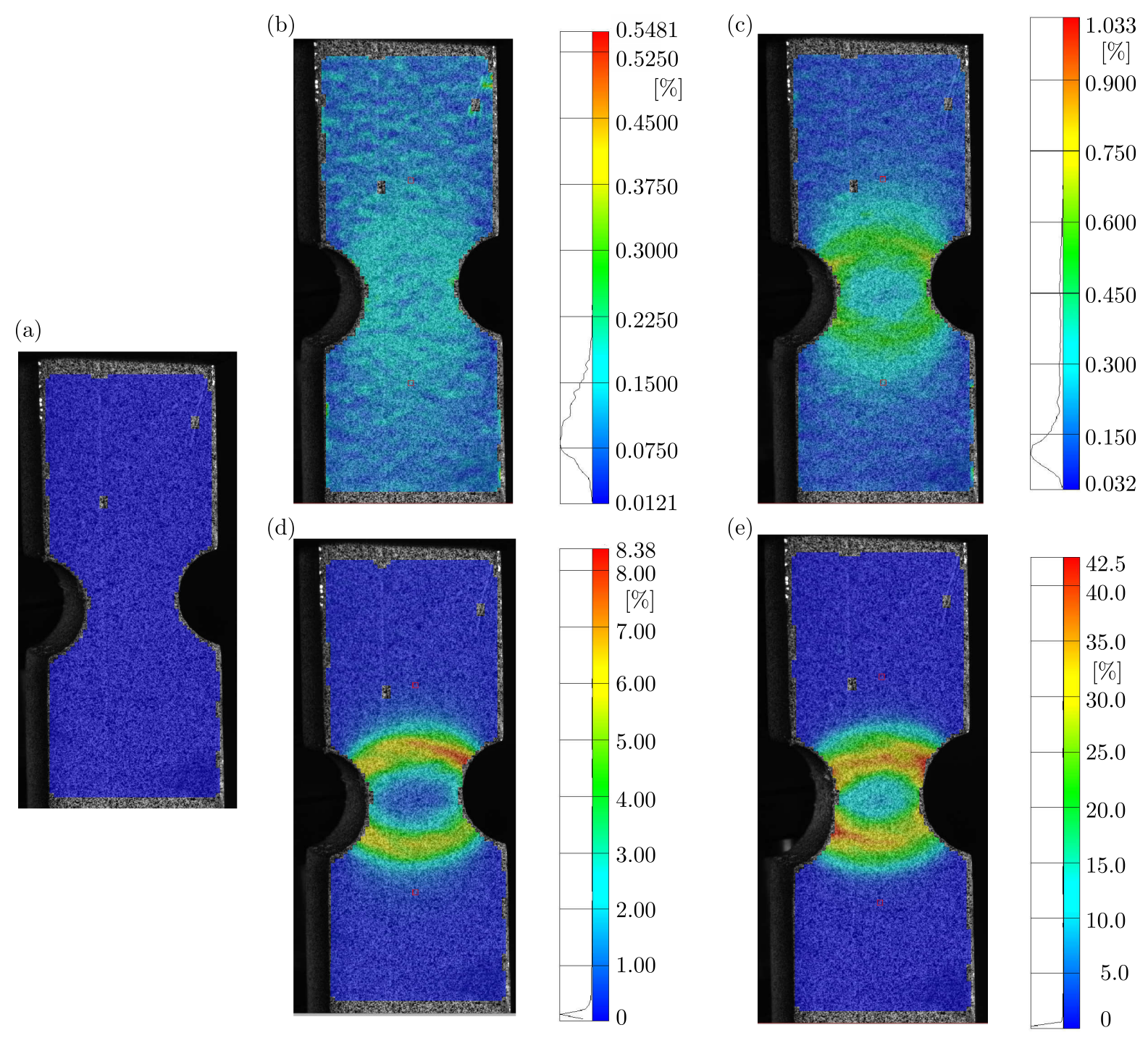

Fig. 10. The equivalent strain distribution in the $U$-notched specimen; material: aluminium 
These arc-shaped deformation regions appear where the growing damage occurs. It is strongly evidenced in the following stages of specimen tension, presenting the material behaviour before fracture (Figs. 10d,e). As it can be noticed in Figs. 10c-e, the strain distribution is expressed by the zone in form of an arc at the test beginning up to the specimen fracture. It shows that besides the strain concentration, which can be captured in the DIC experiments, the shape of strain should be taken into account as data for further analysis of the effects related to the $U$-shaped notch.

The making use of the DIC system has also been examined in the test on determination of the influence of dimensions of geometrical imperfections in $U$-shaped (Figs. 11 and 12) and $V$-shaped specimens (Figs. 15 and 16) on the material fracture. The depth of the notches was the same and equal to $1.3 \mathrm{~mm}$. The radii of the $U$-notches were of $0.75 \mathrm{~mm}, 1.5 \mathrm{~mm}$ and $2.5 \mathrm{~mm}$. The angles between the edges of the $V$-notches were $30^{\circ}, 60^{\circ}$ and $90^{\circ}$, respectively.

(a)

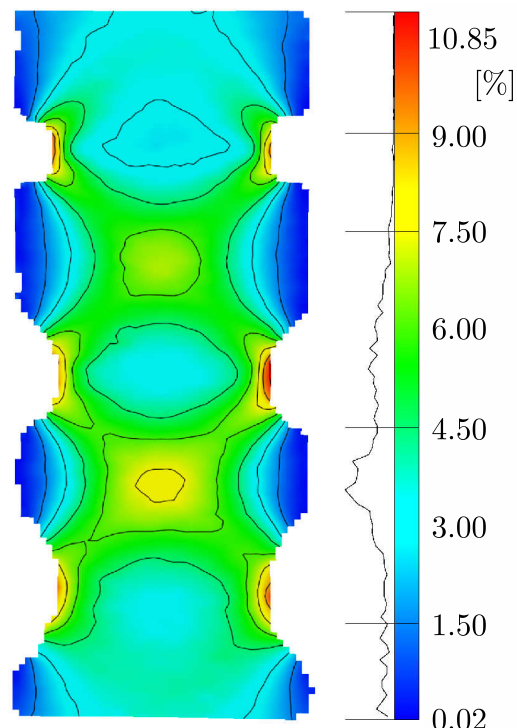

(c)

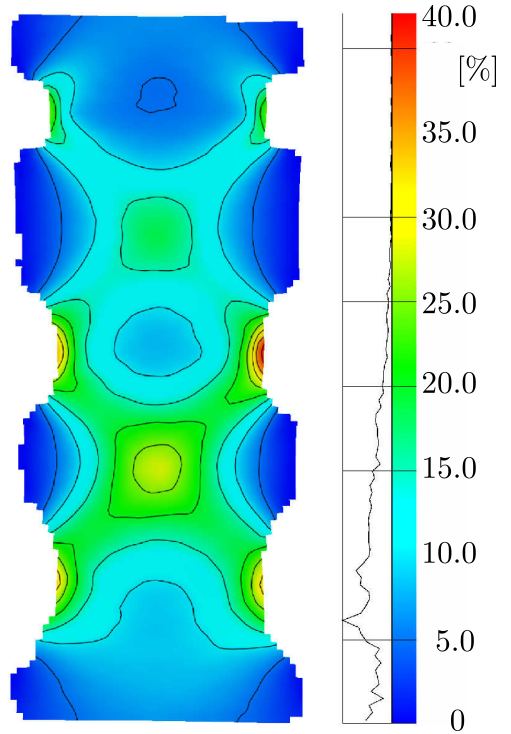

(b)

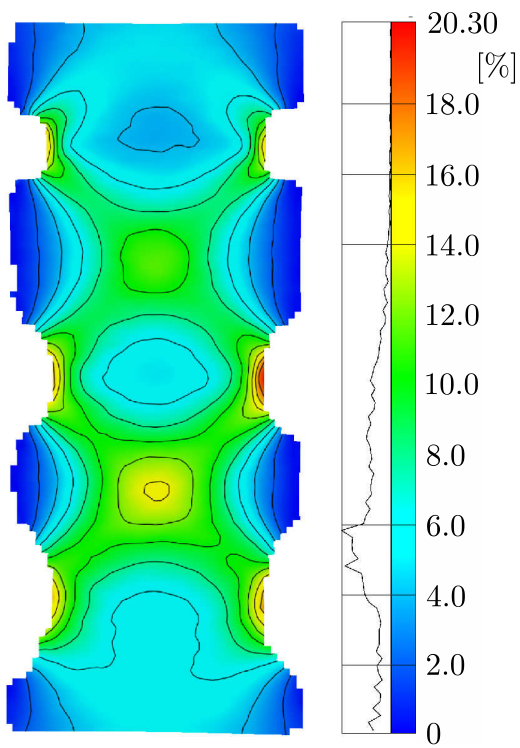

(d)

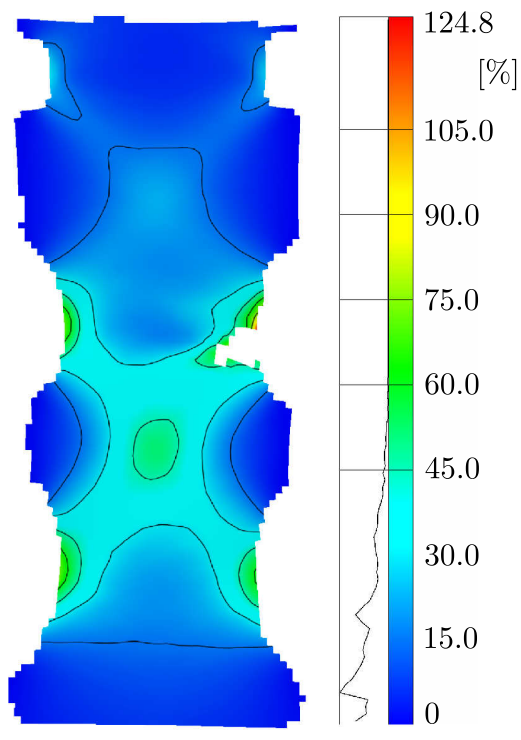

Fig. 11. The equivalent strain distribution in the $U$-shaped multi-notched specimen at various stages of monotonic tension: (a), (b), (c) elastic-plastic state, (d) elastic-plastic state before fracture; material: the $41 \mathrm{Cr} 4$ steel 

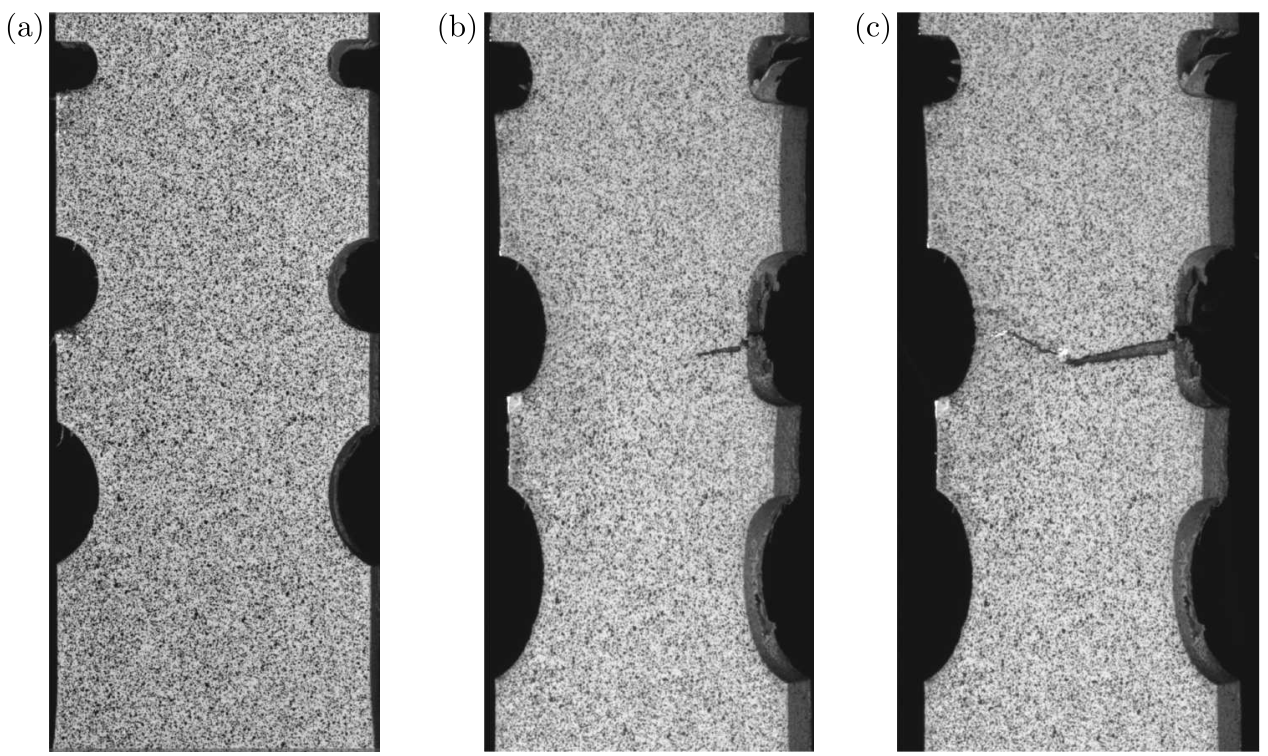

Fig. 12. Three stages of the $U$-notched specimen during monotonic tension: (a) before loading,

(b) crack appearing, (c) crack growing

Effects resulting from the dimensions of the $U$ and $V$ notches have been determined based on variations of the equivalent strain isolines (Figs. 11 and 15). In the case of the $U$ multi-notched specimen, the interaction between the notches became more significant with an increase of theirs radius (Fig. 11b). The results achieved for further tension also expressed that effect (Fig. 11c). It disappeared when the damage zone became greater (Fig. 11d), and it vanished with growing of the cracks. The cracks can also be followed on the basis of typical photos from the DIC of CCD camera (Fig. 12). This is very important for the final stage of testing, because crack growing causes a fracturing measurement pattern and, therefore, the digital correlation is not possible to be done (Figs. 11d and 12b,c).

Assessment of the effects of notches has been followed by using analytical equations for the stress concentration factor and the maximum stress (Pilkey, 1997). Their form for the multi-notched $U$ specimens is noted in Eqs. (3.2), where $K_{t U}$ is the stress concentration factor, $r$ is the notch radius, $d$ is the distance between the centre of opposite notches, $D$ is specimen width, and $L$ is the distance between the notches

$$
\begin{aligned}
& K_{t U}=C_{1}+C_{2} \frac{2 r}{L}+C_{3}\left(\frac{2 r}{L}\right)^{2}+C_{4}\left(\frac{2 r}{L}\right)^{3} \\
& C_{1}=3.1055-3.4287 \frac{2 r}{D}+0.8522\left(\frac{2 r}{D}\right)^{2} \\
& C_{2}=-1.4370-10.5053 \frac{2 r}{D}-8.7547\left(\frac{2 r}{D}\right)^{2}-19.6237\left(\frac{2 r}{D}\right)^{3} \\
& C_{3}=-1.6753-14.0851 \frac{2 r}{D}+43.6575\left(\frac{2 r}{D}\right)^{2} \\
& C_{4}=1.7207+5.7974 \frac{2 r}{D}-27.7463\left(\frac{2 r}{D}\right)^{2}+6.0444\left(\frac{2 r}{D}\right)^{3}
\end{aligned}
$$

Variations of the stress concentration factor versus notch radius express a linear reduction of its value with the radius increase, Fig. 14a. The same course is noticed for the maximum stress, as can be seen in Fig. 14b. This magnitude has been reached applying the following formula

$$
\sigma_{\max }=K_{t U} \sigma_{n o m}
$$

where $\sigma_{\text {nom }}=F / A_{0}$ is the nominal stress, $A_{0}$ - area of the specimen cross section for the measurement zone. 


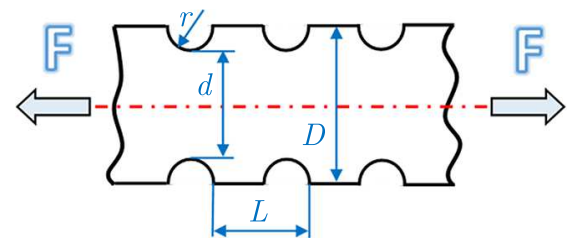

Fig. 13. $U$ multi-notched specimen with the dimensions (Pilkey, 1997)
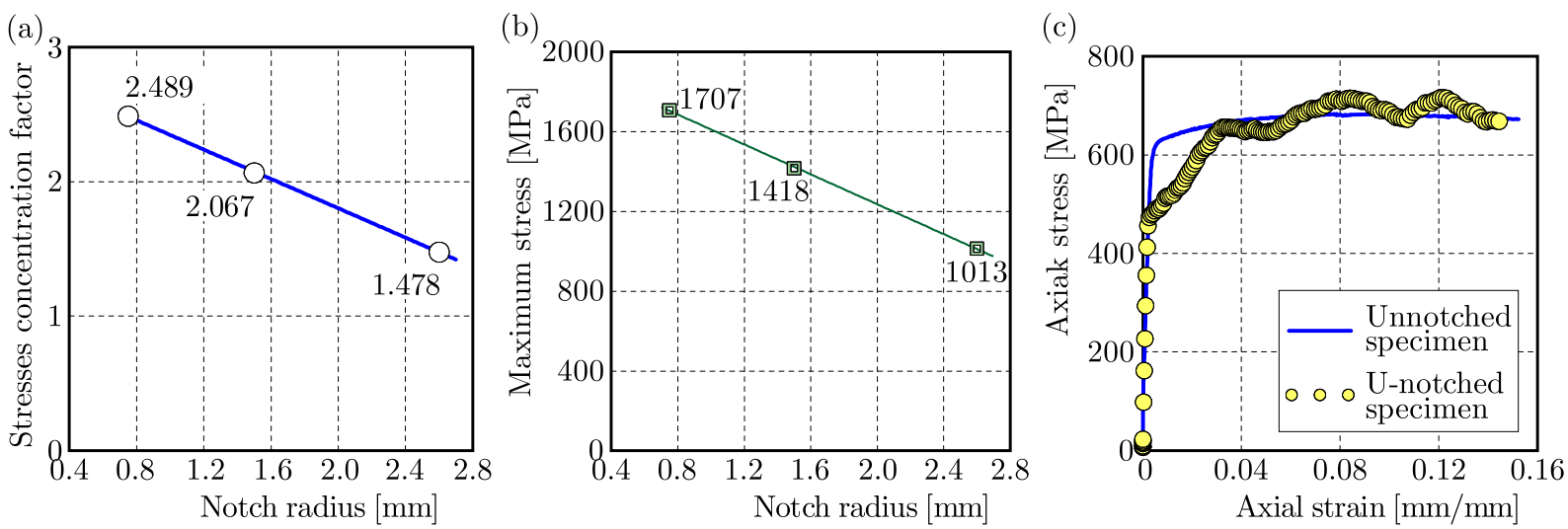

Fig. 14. Results for the multi-notched $U$ specimen: (a) and (b) stress concentration factor and maximum stress as a function of notch radius, respectively, (c) tensile curve variations calculated by employing the cross section of the specimen

Looking at these results, it is easy to indicate the notches which are the dominant geometrical imperfections for the crack appearance, i.e. having a radius of $0.75 \mathrm{~mm}$. This fact has not been confirmed by the DIC results in the final stages of tension (Figs. 11c,d), where the main crack occurred in the middle notch, for which the calculated maximum stress was lower (close to $300 \mathrm{MPa}$ ) than for the smallest radius considered.

The effects resulting from the presence of $V$-notches can also be captured by means of the DIC (Fig. 15). For this type of geometrical imperfections, the full-field equivalent strain distributions close to the tip of the notches and the entire measurement section can be observed (Fig. 15b). Besides different values of the $V$-notch angle, the indication of the main concentrator for the maximum strain is difficult. The $30^{\circ}$ and $60^{\circ}$ notches appear to have a very similar influence on the strain distribution at the beginning of the test. Further tension leads to an increase of the strain level in both notches diagonal arrangement which appear to be geometrical imperfections for the main damage zones (Figs. 15c, 16b). In these sections, the equivalent strain increases, causing a fracture in the middle notch and then in the diagonal one (Figs. 15d, 16c).

For this type of geometrical imperfections, the stress concentration factor and the maximum stress have been calculated by the use of the following formulas (Pilkey, 1997)

$$
\begin{aligned}
& K_{t V}=C_{1}+C_{2} \sqrt{K_{t U}}+C_{3} K_{t U} \\
& C_{1}=-10.01+0.1534 \alpha-0.000647 \alpha^{2} \\
& C_{2}=13.60-0.2140 \alpha+0.000973 \alpha^{2} \\
& C_{3}=-3.781+0.07873 \alpha-0.000392 \alpha^{2}
\end{aligned}
$$

where $K_{t V}, K_{t U}$ are the stress concentration factors for the $V$ and $U$ notches, respectively; $C_{i}$ are coefficients, and $\alpha$ is the angle between the edges of the notches. The maximum stress is found 
(a)

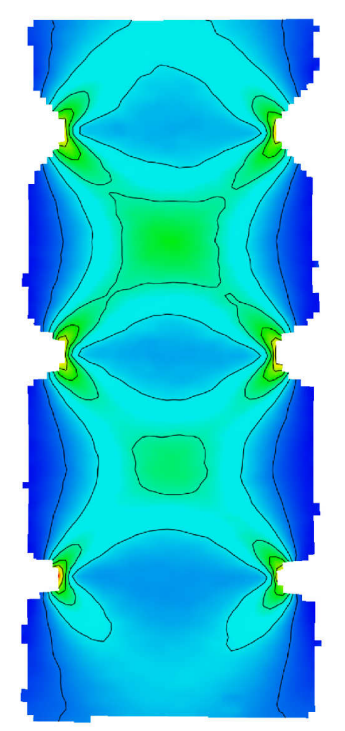

(c)

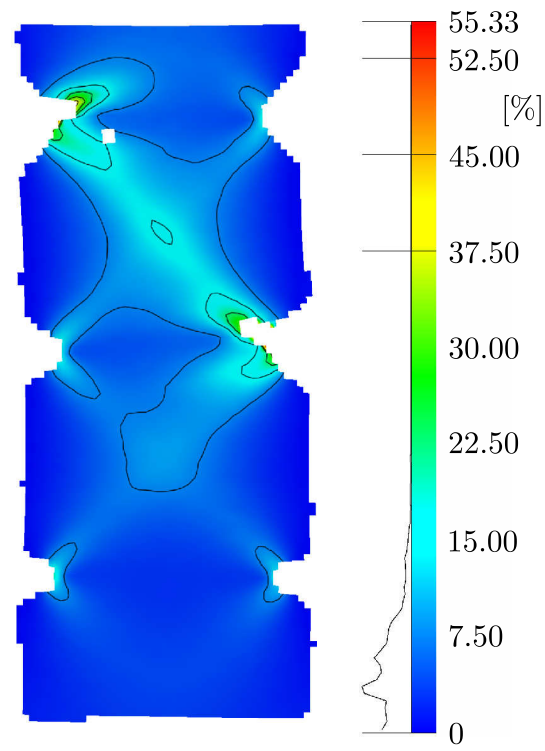

(b)

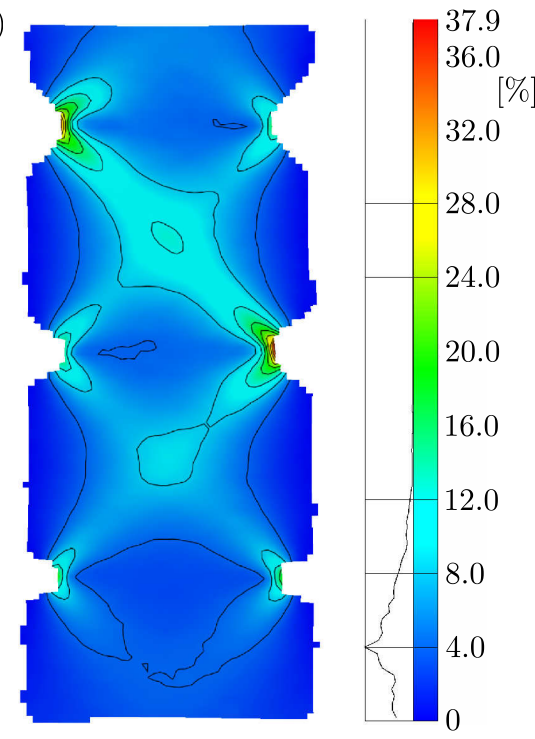

(d)

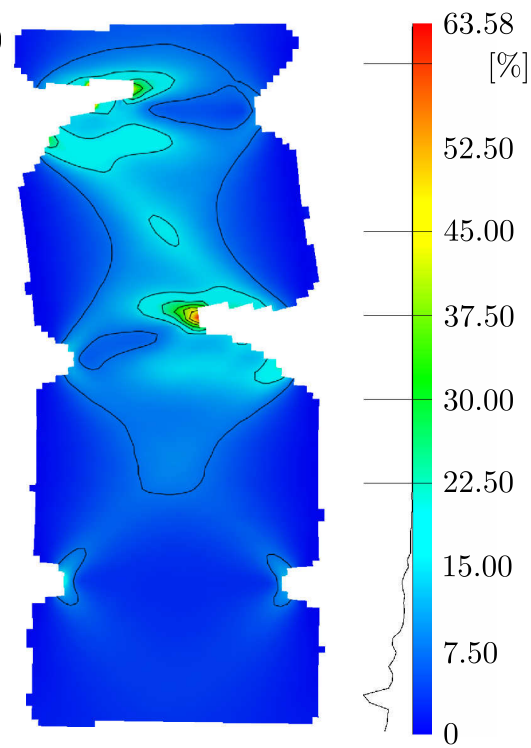

Fig. 15. The equivalent strain distribution in the multi-notched $V$ specimen at various stages of monotonic tension: (a), (b) elastic-plastic state, (c), (d) elastic-plastic state before fracture; material: the $41 \mathrm{Cr} 4$ steel

applying the following relationship $\sigma_{\max }=K_{t V} \sigma_{n o m}$. The results express small lowering of the stress concentration factor and the maximum stress with the decreasing notch angle, Fig. 17. These data strongly correspond with the final stage of tension of the multi-notched $V$ specimen (Figs. 15c,d). Small differences between the values of the stress concentration factor and the maximum stress at various notch angles show that all of the examined $V$ notches are also the places where the damage zones can occur.

The influence of the $V$-notch on material straining during tension is illustrated in Fig. 17c presenting a comparison of tensile curves determined by the use of smooth and $V$ multi-notched specimens. It is easy to notice that the presence of this type geometrical imperfection caused a $50 \%$ reduction of elongation and $30 \%$ lowering of the yield point. 
(a)

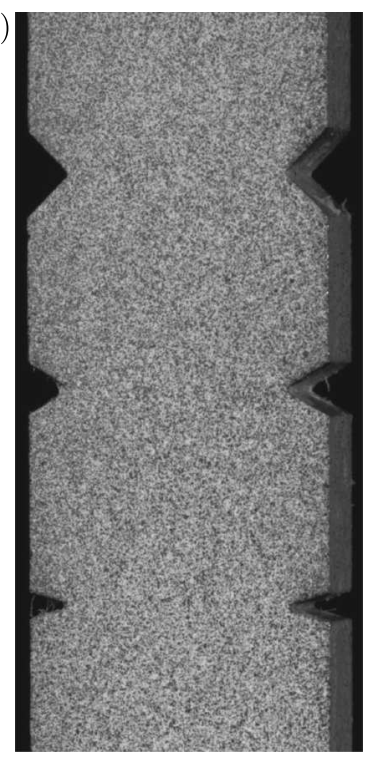

(b)

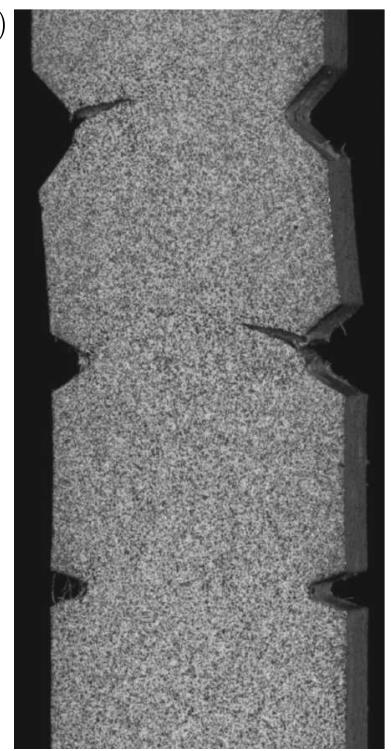

(c)

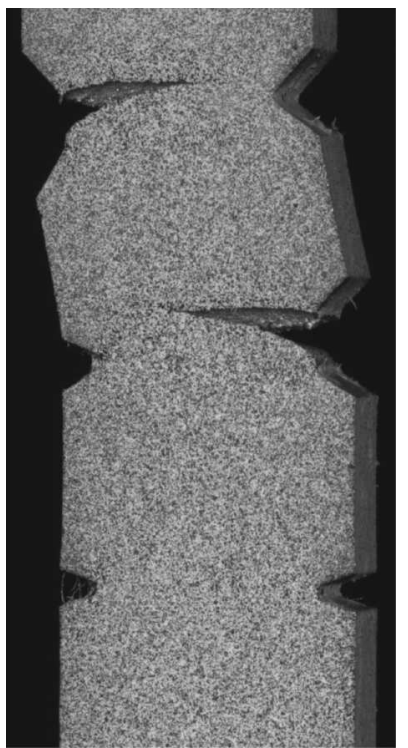

Fig. 16. Three stages of the multi-notched $V$ specimen during monotonic tension: (a) before loading, (b) crack initiation, (c) crack growth
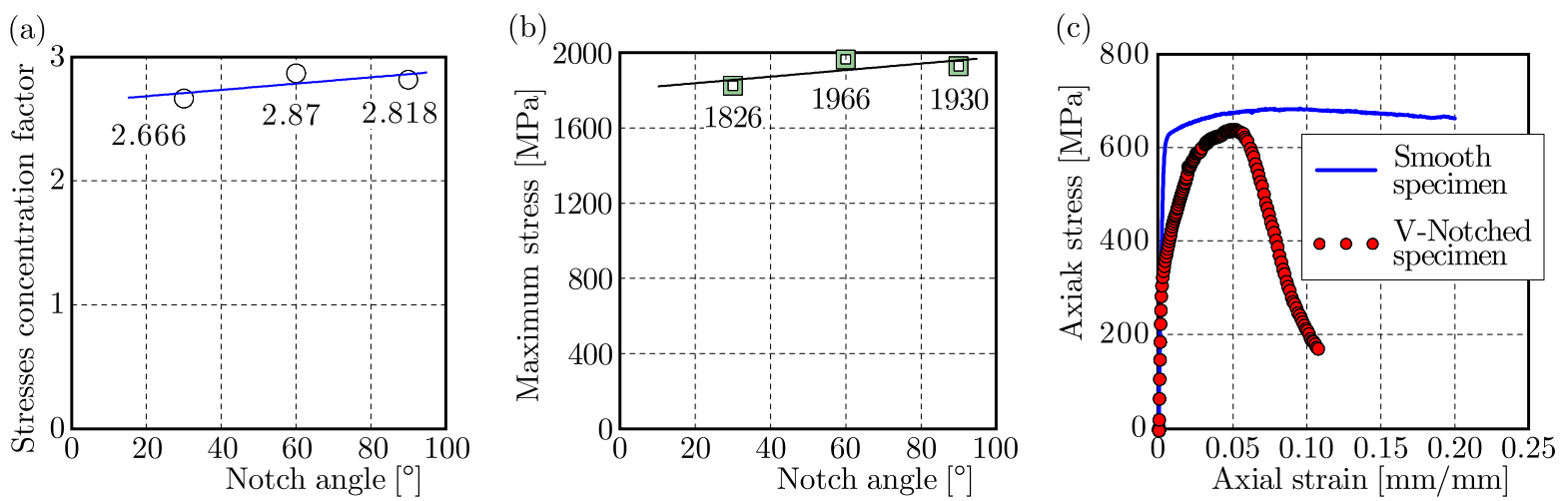

Fig. 17. Results for the multi-notched $V$ specimen: (a) and (b) stress concentration factor and the maximum stress as a function of notch radius for the $V$-notched specimen, (c) tensile curve variations with respect to dimension of the cross section of the specimen; respectively

\section{Summary}

The Digital Image Correlation method can be employed for tests on specimens with or without geometrical imperfections. This technique allows capturing damage zones up to specimen fracture.

The DIC method follows the interaction of strain fields resulting from the presence of notches. Isolines created in a multi-notched specimen occur at the initial stage of tension and disappear at the moment of damage zones focusing and crack appearing.

Independently of the type of geometrical imperfections considered, a significant reduction of the proportional limit and yield point has been observed.

A typical DIC device is able to reach strain distribution close to the notch in the elastic-plastic state. In the case of the elastic state, the micro-DIC method using a microscope device is more recommended.

The Digital Image Correlation technique can be successfully used for determination of the Young's modulus, yield point, ultimate tensile strength and elongation. 


\section{Acknowledgement}

The paper was partly carried out in the framework of the project No. 2014/15/B/ST8/04368 founded by the National Science Centre.

\section{References}

1. Andersson M., 2013, The influence of notches on fatigue of heat treated sintered steel, EURO PM 2013, Gothenburg, 6 p.

2. Bennett J.A., Weinberg J.G., 1954, Fatigue notch sensitivity of some aluminium alloys, Journal of Research of the National Bureau of Standards, 52, 5, 235-245

3. Boroński D., 2007, Methods for Examination of Strain and Stress under Fatigue of Material and Structures (in Polish), Institute for Sustainable Technologies, National Research Institute, Bydgoszcz-Radom, Poland, $190 \mathrm{p}$.

4. Chu T.C., Ranson W.F., Sutton M.A, Peters W.H., 1985, Application of digital-image-correlation techniques to experimental mechanics, Experimental Mechanics, 25, 3, 232-244

5. Chen C., 2004, General theory, ME631, UAF, 15 p.

6. Da Silva B.L., Ferreira J.L.A., Arajo J.A., 2012, Influence of notch geometry on the estimation of the stress intensity factor threshold by considering the Theory of Critical Distances, International Journal of Fatigue, 42, 258-270

7. DuQuensay D.L., Topper T.H., Yu M.T., 1986, The effect of notch radius on the fatigue notch factor and the propagation of short cracks, [In:] The Behaviour of Short Fatigue Cracks, Edited by K.J. Miller and E.R. de los Rios, EGF Pub., Mechanical Engineering Publications, London, 323-335

8. Durif E., Réthoré J., Combescure A., Fregonese M., Chaudet P., 2012, Controlling stress intensity factors during a fatigue crack propagation using digital image correlation and a load shedding procedure, Experimental Mechanics, 52, 8, 1021-1031

9. Fatemi A., Fang D., Zeng Z., 2002, Notched fatigue behaviour under axial and torsion loads: experiment and predictions, 8th International Fatigue Congress, Stockholm, Sweden, 3, 1905-1914

10. Fatemi A., Zeng Z., Plaseied A., 2004, Fatigue behaviour and life predictions on notched specimens made of QT and forged microalloyed steels, International Journal of Fatigue, 26, 6, $663-672$

11. Filippini M., 2000, Stress gradient calculations at notches, International Journal of Fatigue, 22, 5, 397-409

12. Forster J., Theobald A., Engel S., Pasmann R., 2012, Using optical measuring system for identification of material parameters for Finite Element Analysis, 11. LS-DYNA, Ulm, Germany, $9 \mathrm{p}$.

13. Gower M.R., Shaw R.M., 2010, Towards a planar cruciform specimen for biaxial characterization of polymer matrix composites, Applied Mechanics and Materials, 24-25, 115-120

14. KamaYA M., KAWAKUBO M., 2011, A procedure for determining the true stress-strain curve over a large range of strains using digital image correlation and finite element analysis, Mechanics of Materials, 43, 5, 243-253

15. Lanning D.B., Haritos G.K., Nicholas T., 1999, Influence of stress state on high cycle fatigue of notched Ti-6Al-4V specimens, International Journal of Fatigue, 21, 1, S87-S95

16. LorD J.D., 2009, Digital Image Correlation (DIC), Modern stress and strain analysis, [In:] A State of the Art Guide to Measurement Techniques, BSSM Technical Editors: J. Eaton Evans, J.M. Dulie-Barton, R.L. Burguete, 14-15

17. Maruno Y., Miyahara H., Noguchi H. and Ogi K., 2004, Notch size effects in the fatigue characteristics of Al-Si-Cu-Mg cast alloy, Materials Transactions, 45, 3, 839-843 
18. Mazdumar P.K., Lawrence F.V. JR., 1981, An analytical study of the fatigue notch size effect, A Report of the Fracture Control Program, College of Engineering, University of Illinois, Urbana, Illinois 6180

19. Milke J.G., Beuth J.L., Biry N.E., 2000, Notch strengthening in titanium alumini dies under monotonic loading, Experimental Mechanics, 4, 4, 415-424

20. Neuber H., 1958, Kerbspannungslehre, 2nd ed., Berlin, Springer-Verlag

21. Neuber H., 1961, Theory of Notch Stresses, Office of Technical Services, U.S. Department of Commerce, Washington, DC

22. Olszak W., 1965, Theory of Plasticity, PWN, Warsaw

23. Peterson R.E., 1959, Analytical approach to stress concentration effect in fatigue of aircraft materials, Proceedings on Fatigue of Aircraft Structure, WADC Technical Report No. 59-507, August 1959, 273-299

24. Pilkey W.D., 1997, Peterson's Stress Concentration Factors, Wiley, New York

25. Pluvingae G., Guonaj M., 2001, Notch Effects in Fatigue and Fracture, NATO Science Series, Series II: Mathematics, Physics and Chemistry, 11, Springer Science+Business Media, B.V.

26. Qian G., Hong Y., Zhou Ch., 2010, Investigation of high cycle and very-high-cycle fatigue behaviors for a structural steel with smooth and notched specimens, Engineering Failure Analysis, 17, 7-8, 1517-1525

27. Siebel E., Stieler M., 1955, Ungleichformige spannungsverteilung bei schwingender beanspruchung, Zeitschrift des Vereines Deutscher Ingenieure, VDI-Z, 97, 5, 121-126

28. Topper T.H., Wetzel R.M., Morrow J., 1967, Nuber's rule applied to fatigue of notched specimens, Report No. NAEC-ASL-1114, Aeronautical Structures Laboratory, 15 p.

29. Toussaint F., Tabourot L., Vacher P., 2008, Experimental study with a Digital Image Correlation (DIC) method and numerical simulation of an anisotropic elastic-plastic commercially pure titanium, Archives of Civil and Mechanical Engineering, 8, 3, 131-143

30. Wahl A.M., Beuwkes R., Pittsburgh E., 1930, Stress concentration produced by holes and notches, Applied Mechanics, 56, 11, 617-623

31. Westergand H.M., 1952, Theory of Elasticity and Plasticity, New York, John Wiley

32. Whaley R.E., 1964, Fatigue and static strength of notched and un-notched aluminium alloy and steel specimens, Experimental Mechanics, 329-334

33. www.gom.com 